MODELING, IDENTIFICATION AND CONTROL, 1983, VOL. 4, NO. 1, 47-62

doi:10.4173/mic.1983.14

\title{
Disturbance error reduction in multivariable optimal control systems $\dagger$
}

\author{
OLE A. SOLHEIM $\ddagger$ and ULF STENHAUG $\ddagger$
}

Keywords: Multivariable control systems, optimal control, state estimation, feedback, feedforward, frequence response.

The paper deals with the design of optimal multivariable controllers, using a modified LQR approach. All controllers discussed contain proportional feedback and, in addition, there may be feedforward, integral action or state estimation.

The disturbance reduction properties of the systems arrived at are evaluated and compared, using frequency response curves for the different output variables, together with singular value plots.

The investigations are based on the use of the CAD-systems DAREK and CYPROS.

\section{Introduction}

One of the most important tasks of a control system is to reduce the effects of disturbances on the system's outputs. The effectiveness of the controller in reducing disturbance errors is therefore an important design criterion. A complete rejection of the effect of disturbances on the output variables is, in some cases, possible and a suitable controller may be designed via the geometric approach (Wonham 1979, Takamatsu, Hashimoto and Nakai 1979, Kümmel and Ohrt 1981). Although this approach may work well in special cases, it also has some rather severe drawbacks. For example, the design procedure is not straightforward and it is difficult to include other design parameters such as optimality and robustness.

Another possibility is to use frequency domain methods, eventually combined with linear quadratic methods (MacFarlane 1981, Doyle and Stein 1981, a number of articles in I.E.E.E. Transactions, AC-26, February 1981, special issue on linear multivariable control systems). The design of multivariable systems in the frequency domain is, however, rather complicated, at least compared with linear quadratic methods.

The purpose of the present paper is to demonstrate the use of a modified LQR (Linear Quadratic Regulator) approach in the design of multivariable controllers, and to show how the disturbance error reduction properties can be visualized using frequency analysis.

\section{Problem statement}

Let

$$
\left.\begin{array}{l}
\dot{x}=A x+B u+C v \\
y=D x
\end{array}\right\}
$$

Received 9 December 1982.

$\uparrow$ Paper presented at the IFAC Symposium 'Computer Aided Design of Multivariable Technological Systems', Purdue University, Lafayette, Indiana, U.S.A., 15-17 September 1982. Printed with the permission of IFAC.

$\$$ The University of Trondheim, The Norwegian Institute of Technology, Division of Engineering Cybernetics, 7034 Trondheim-NTH. 
represent a linear time invariant dynamic system, where $x, u, y$ represent as usual the state, the control input and the output, and $v$ represents process disturbances. In addition there may be sensor noise and disturbances that act directly upon the output $y$, but these types will not be considered here.

A quadratic performance criterion will be used

$$
\begin{aligned}
J & =\frac{1}{2} \int_{0}^{\infty}\left[y^{T} Q_{y} y+u^{T} P u\right] d t \\
& =\frac{1}{2} \int_{0}^{\infty}\left[x^{T} Q x+u^{T} P u\right] d t
\end{aligned}
$$

where

$$
Q=D^{T} Q_{y} D
$$

and where we have assumed that the reference value $y_{\text {ref }}=0$. The reason for using this performance criterion is to keep the output variables close to zero under the influence of process disturbances and, at the same time, to economize with the control effort.

To evaluate the disturbance reduction properties of the system arrived at, we may look at the transfer matrix $H_{y v}(s)$ between disturbances $v$ and output $y$

$$
y(s)=H_{y p}(s) v(s)
$$

We may for example display the frequency response curves $\left|y_{i}(j \omega) / v_{j}(j \omega)\right|$, these giving a good indication of how one particular disturbance acts upon one particular output variable. It is also possible to have a more general evaluation, for example by using some measure of the 'size' of the transfer matrix. The measure we shall use here is the maximum singular value $\bar{\sigma}(s)$, which is identical to the Euclidian norm, that is

$$
\bar{\sigma}(\omega)=\sqrt{ }\left[\lambda_{\max }\left(H^{*}(j \omega) H(j \omega)\right)\right]
$$

In addition to that $\bar{\sigma}(\omega)$ indicates an overall performance, it may also be taken as the 'worst case' response, since

$$
\bar{\sigma}(\omega) \geqslant\left|y_{i}(j \omega) / v_{j}(j \omega)\right|
$$

This relation also reveals a serious drawback using $\bar{\sigma}(\omega)$, namely that if, for example, all $\left|y_{i}(j \omega) / v_{j}(j \omega)\right|$ except one, are very small, then it will be this larger one that determines $\bar{\sigma}(\omega)$. We shall demonstrate this fact below in $\S 5$. One problem that occurs using the quadratic criterion (2) is the choice of the weighting matrices $Q_{y}$ (output) and $P$ (controls). Let us assume that $P$ is chosen according to the 'price' of the different control variables. We may then choose $Q_{y}$ according to which weight we place on keeping the different output variables close to zero. There is, however, the problem that with the chosen $Q_{y}$ the system may not have sufficient stability margin, although we know that the system has absolute stability.

This problem may be solved using a method developed by Solheim $(1972,1980)$ whereby it is possible to modify the original $Q$-matrix so that desired stability properties may be achieved. This is why we stated in the introduction that a modified LQR approach is to be used. In order to simplify the computation of the frequency response curves, we may put the different controller configurations into a general framework. To this end we shall use the following standard form of the transfer matrix $H_{y v}(s)$. throughout the paper

$$
H_{y v}(s)=\tilde{D}(s I-\tilde{A})^{-1} \tilde{C}
$$


where the tilde $\sim$ indicates modified matrices corresponding to the actual configuration.

For the open loop case the transfer matrix is simply

$$
H_{y v}(s)=D(s I-A)^{-1} C
$$

We shall now proceed to a detailed discussion of four possible controller configurations.

\section{Proportional feedback}

See Fig. 1.

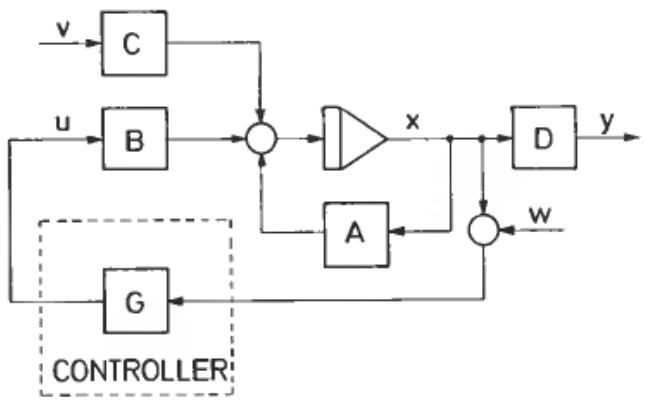

Figure 1. Proportional feedback.

We use the control law

$$
u=G x
$$

where $G=-P^{-1} B^{T} R$ and $R$ is the solution of the usual Riccati equation. The transfer matrix becomes

Thus, in eqn. (6)

$$
H_{y v}(s)=D[s I-(A+B G)]^{-1} C
$$

$$
\tilde{D}=D, \quad \tilde{A}=A+B G, \quad \tilde{C}=C
$$

To illustrate the behaviour of the different controller configurations, we shall use the same simple process throughout the paper:

$$
\begin{aligned}
& A=\left[\begin{array}{rrr}
-2 & 0 & 0 \\
1 & -1 & -1 \\
0 & 10 & -5
\end{array}\right], \quad B=\left[\begin{array}{ll}
1 & 0 \\
0 & 1 \\
0 & 0
\end{array}\right], \\
& D=\left[\begin{array}{lll}
0 & 1 & 0 \\
0 & 0 & 1
\end{array}\right], \quad C=I_{3}, \quad P=\left[\begin{array}{ll}
1 & 0 \\
0 & 5
\end{array}\right]
\end{aligned}
$$

\section{Example 1}

We place equal weight on the two output variables $y_{1}$ and $y_{2}$

$$
Q_{y}=\left[\begin{array}{rr}
100 & 0 \\
0 & 100
\end{array}\right] \text { or } Q=D^{T} Q_{y} D=\left[\begin{array}{rrr}
0 & 0 & 0 \\
0 & 100 & 0 \\
0 & 0 & 100
\end{array}\right]
$$


The eigenvalues of the closed loop system $(A+B G)$ then become

$$
-2 \cdot 92,-5 \cdot 62 \pm j 4 \cdot 56
$$

The eigenvalue at -2.92 is too close to the imaginary axis, so we choose to move it to -8 . This can be achieved through the modified $Q$-matrix

$$
Q=\left[\begin{array}{rcc}
55.09 & -10.32 & 12.04 \\
-10.32 & 101.9 & -2.26 \\
12.04 & -2.26 & 102.6
\end{array}\right]
$$

The feedback matrix becomes

$$
G=\left[\begin{array}{lll}
-5.88 & -1.88 & -1.03 \\
-0.38 & -5.36 & -0.98
\end{array}\right]
$$

We compute $\left|y_{i}(j \omega) / v_{j}(j \omega)\right|, i=1,2, j=1,2,3$. The result is shown in Fig. 2. This figure also shows the $\bar{\sigma}$-plot.

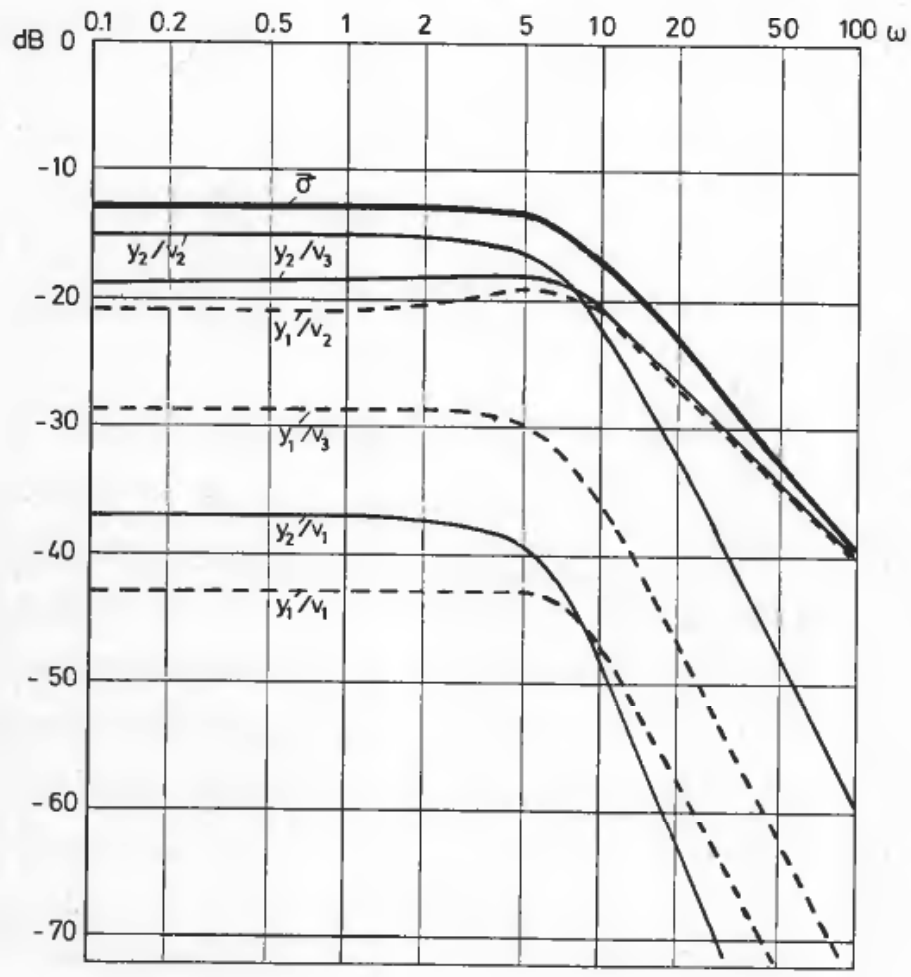

Figure 2. Example 1.

If the performance of this system is not satisfactory, there are several possibilities for improvement, as for example increasing the elements of the $Q_{y}$-matrix, introducing feedforward or adding integral action. All these measures will be considered in the following. 


\section{Example 2}

Let us start with increasing the weight on the output variables. Let

$$
Q_{y}=\left[\begin{array}{rr}
1000 & 0 \\
0 & 1000
\end{array}\right] \text { or } Q=\left[\begin{array}{rrr}
0 & 0 & 0 \\
0 & 1000 & 0 \\
0 & 0 & 1000
\end{array}\right]
$$

We check the eigenvalues:

$$
-2 \cdot 99,-11 \cdot 39 \pm j 5 \cdot 403
$$

We note that this new $Q$-matrix does not change very much the eigenvalue closest to the imaginary axis. We again shift this eigenvalue to -8 through the modified $Q$-matrix:

$$
Q=\left[\begin{array}{ccc}
54.95 & -5.38 & 14.64 \\
-5.38 & 1001 & -1.43 \\
14.64 & -1.43 & 1004
\end{array}\right]
$$

The feedback matrix becomes

$$
G=\left[\begin{array}{lll}
-5.97 & -3.62 & -1.48 \\
-0.725 & -16.8 & -5.93
\end{array}\right]
$$

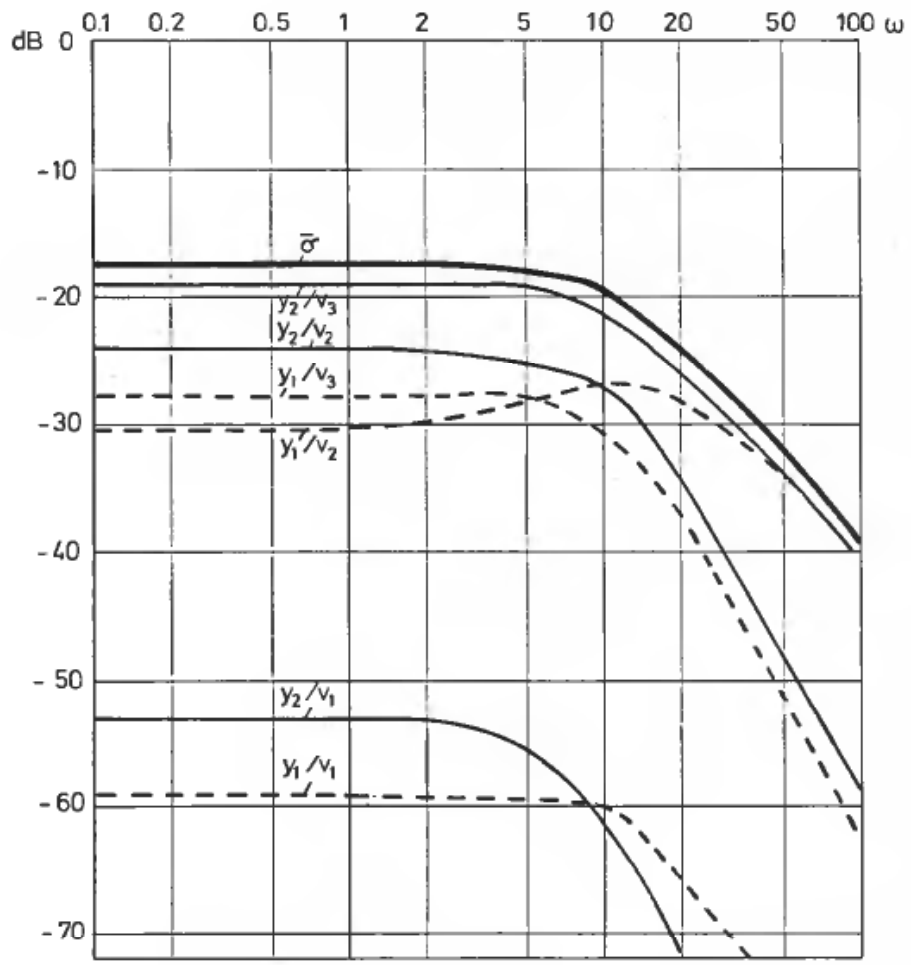

Figure 3. Example 2. 
The frequency response curves together with the $\bar{\sigma}$-plot are shown in Fig. 3. We note that a considerable improvement has been achieved for most of the curves but very little, for example, for $y_{2} / v_{3}$. The general improvement is also indicated by the $\bar{\sigma}$-plot.

\section{Proportional feedback and feedforward}

Assuming that the disturbances can be measured, we may introduce a feedforward control matrix $G_{2}$ in addition to the feedback control matrix $G_{1}$, Fig. 4. In order to achieve exact optimal control, it is necessary to know the future disturbances. If we assume constant disturbances, the situation becomes simple, since the optimal solution in this case is a constant feedforward matrix given by

$$
G_{2}=P^{-1} B^{T}\left(\left(A+B G_{1}\right)^{-1}\right)^{T} R C
$$

where $R$ again is the solution of the usual Riccati equation and $G_{1}$ is the same feedback matrix as in (8).

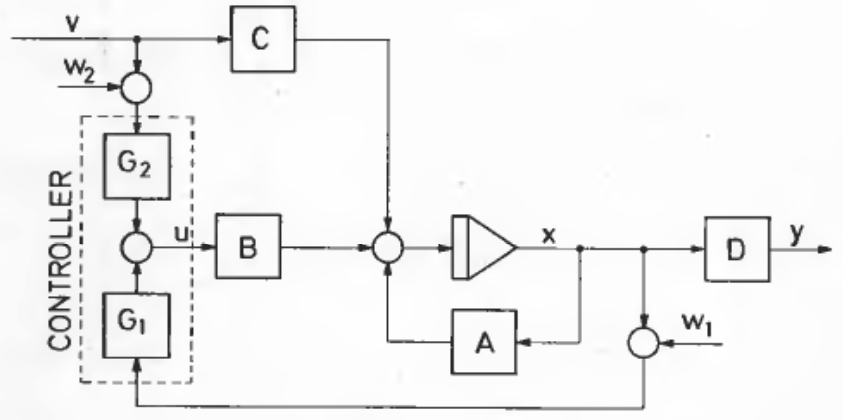

Figure 4. Proportional feedback and feedforward.

If the disturbances are constant or slowly varying (slowly relative to the process dynamics) we should expect that the disturbance reduction properties are improved compared to the feedback-only case. For more rapidly varying disturbances the situation could be different.

We get the transfer matrix

$$
H_{y v}(s)=D\left[s I-\left(A+B G_{1}\right)\right]^{-1}\left(B G_{2}+C\right)
$$

The matrices used in the standard form (6) will then be

$$
\tilde{D}=D, \quad \tilde{A}=A+B G_{1}, \quad \tilde{C}=B G_{2}+C
$$

\section{Example 3}

We use the same $Q$-matrix as in Example 1, and thus the feedback matrix will be the same. The feedforward matrix becomes

$$
G_{2}=\left[\begin{array}{ccc}
-0.754 & -0.504 & -0.293 \\
-0.0188 & -0.671 & -0.410
\end{array}\right]
$$

The frequency response curves and $\bar{\sigma}$-plot for this example are shown in Fig. 5. Comparing these curves with those of Fig. 2 (Example 1), we note that a considerable 


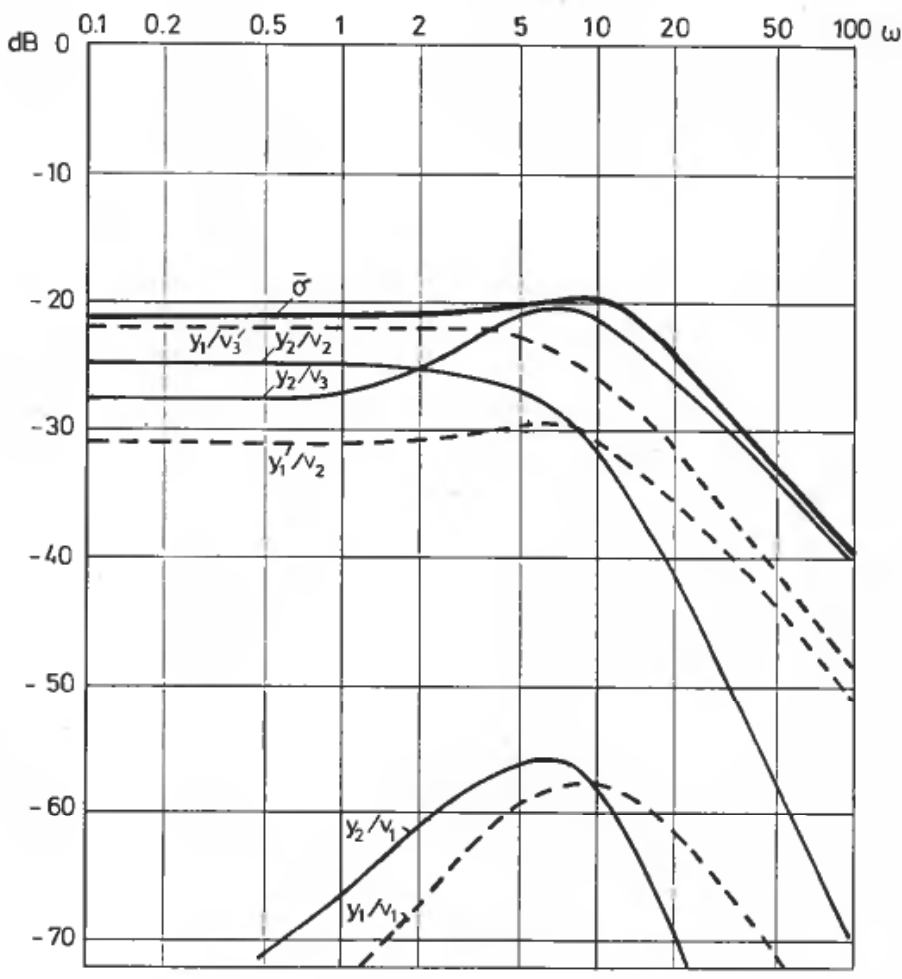

Figure 5. Example 3.

improvement has been obtained except for $y_{1} / v_{3}$ which is better with feedback only. This is, however, what we must expect in a multivariable system. The overall situation may improve, as is also indicated by the $\bar{\sigma}$-plot in this case, but for some of the variables the situation may deteriorate.

\section{Proportional plus integral feedback}

In order to improve the steady-state and low-frequency performance, we may try to include an integral action in the feedback loop, Fig. 6.

The control law becomes in this case:

$$
u=G_{1} x+G_{2} z
$$

where $z$ is the integrator output, described by

$$
\dot{z}=E y=E D x
$$

The $E$-matrix will single out those output variables for which we want to improve the steady-state and low frequency behaviour.

Let us establish the augmented system

$$
\dot{x}=\left[\begin{array}{c}
\dot{x} \\
\hdashline \dot{z}
\end{array}\right]=\left[\begin{array}{c:c}
A & 0 \\
\hdashline E D & 0
\end{array}\right] \tilde{x}+\left[\begin{array}{c}
B \\
\hdashline 0
\end{array}\right] u+\left[\begin{array}{c}
C \\
\hdashline 0
\end{array}\right] v
$$




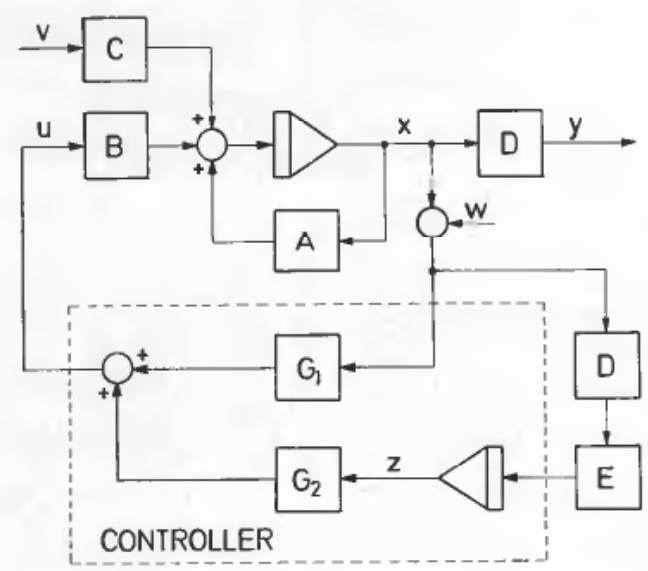

Figure 6. Proportional plus integral feedback.

In order that this system be controllable, the system $(A, B)$ must be controllable and in addition must $\operatorname{dim} z \leqslant \operatorname{dim} u$. There can, therefore, be no more integrators than control variables.

Combining (12) and (14) yields the closed loop system

$$
\begin{aligned}
\hat{x} & =\left[\begin{array}{c:c}
A+B G_{1} & B G_{2} \\
\hdashline E D & 0_{p \times p}
\end{array}\right] \tilde{x}+\left[\begin{array}{c}
C \\
\hdashline 0_{p \times n}
\end{array}\right] v \\
& =\tilde{A} \tilde{x}+\tilde{C} v
\end{aligned}
$$

Here $p=\operatorname{dim} z, n=\operatorname{dim} x$.

From (14) we note that the eigenvalues of the open-loop system consist of the eigenvalues of the process $\mathrm{A}$ and as many eigenvalues at the origin as there are integrators. The effectiveness of the integral action will now depend upon how far to the left we shift these last eigenvalues. Compared with a conventional PI-controller, a large integral time corresponds to a small shift of the eigenvalues.

The matrices in the standard transfer matrix (6) are for this multivariable PIcontroller

$$
\tilde{D}=\left[\begin{array}{ll}
D & 0_{m \times p}
\end{array}\right]
$$

where $m=\operatorname{dim} y$ and $p=\operatorname{dim} z$.

$\tilde{A}$ and $\tilde{C}$ are as given in (15).

\section{Example 4}

We choose $E=\left[\begin{array}{ll}0 & 1\end{array}\right]$ and start with the same $Q_{y}$ as in Example 1. We need an augmented $Q$-matrix:

$$
\tilde{Q}=\left[\begin{array}{rrrr}
0 & 0 & 0 & 0 \\
0 & 100 & 0 & 0 \\
0 & 0 & 100 & 0 \\
0 & 0 & 0 & 0
\end{array}\right]
$$


With this $\tilde{Q}$-matrix we get the following eigenvalues

$$
0, \quad-2 \cdot 92, \quad-5 \cdot 62 \pm j 4 \cdot 56
$$

We choose to move the eigenvalue at -2.92 to -8 , and, starting with a rather weak integral action, we move the eigenvalue at the origin to $-0 \cdot 5$.

The corresponding $\tilde{Q}$-matrix becomes

$$
\tilde{Q}=\left[\begin{array}{cccc}
55.1 & -10.23 & 12.1 & 0.51 \\
-10.23 & 103.1 & -1.47 & 6.45 \\
12.1 & -1.47 & 103.1 & 4.19 \\
0.51 & 6.45 & 4.19 & 34.28
\end{array}\right]
$$

which yield the feedback matrices

$$
\begin{aligned}
& G_{1}=\left[\begin{array}{lll}
-5.9 & -2.07 & -1.15 \\
-0.414 & -5.84 & -1.3
\end{array}\right] \\
& G_{2}=\left[\begin{array}{l}
-1.02 \\
-2.58
\end{array}\right]
\end{aligned}
$$

We should now expect that all $\left|y_{2}(j \omega) / v_{j}(j \omega)\right| \rightarrow(-\infty \mathrm{dB})$ as $\omega \rightarrow 0$. This is also the case but, since the frequence range for the plots starts at $\omega=0 \cdot 1$, this is not quite clear from the frequency response curves, Fig. 7. We see, however, the trend. These curves are to be compared with those in Fig. 2 (Example 1) for the corresponding proportional feedback case. We note that the integral action is effective below $\omega \approx 2$. We note also that for $y_{1} / v_{3}$ the situation has deteriorated. Remembering that $y_{1}$ is not input to an integrator, this is what can be expected.

We also note that the $\bar{\sigma}$-plot in Fig. 7 does not exhibit the shape that we perhaps should expect when using a PI-type controller, although we note some low-frequency improvement comparing with the $\bar{\sigma}$-plot in Fig. 2 . The reason why $\bar{\sigma}$ does not have a typical integral shape $(\rightarrow-\infty \mathrm{dB}$ as $\omega \rightarrow 0)$ is, of course, that there is still a large element in the $H_{y r}(s)$ matrix when $\omega \rightarrow 0$, namely $y_{1} / v_{3}$. This is a weakness in $\bar{\sigma}$-plots and shows the usefulness of individual $y_{i} / v_{j}$ plots.

\section{Example 5}

We start in the same way as in Example 4 and move the eigenvalue a -2.92 at -8 as before, but we will now try a stronger integral action and move the eigenvalue at the origin to -2 . The result is

$$
Q=\left[\begin{array}{cccc}
55 \cdot 2 & -8.8 & 13.04 & 8 \cdot 17 \\
-8.8 & 121 \cdot 3 & 10.35 & 103 \cdot 1 \\
13.04 & 10.35 & 110.8 & 67.06 \\
8.17 & 103.1 & 67.06 & 548.4
\end{array}\right]
$$




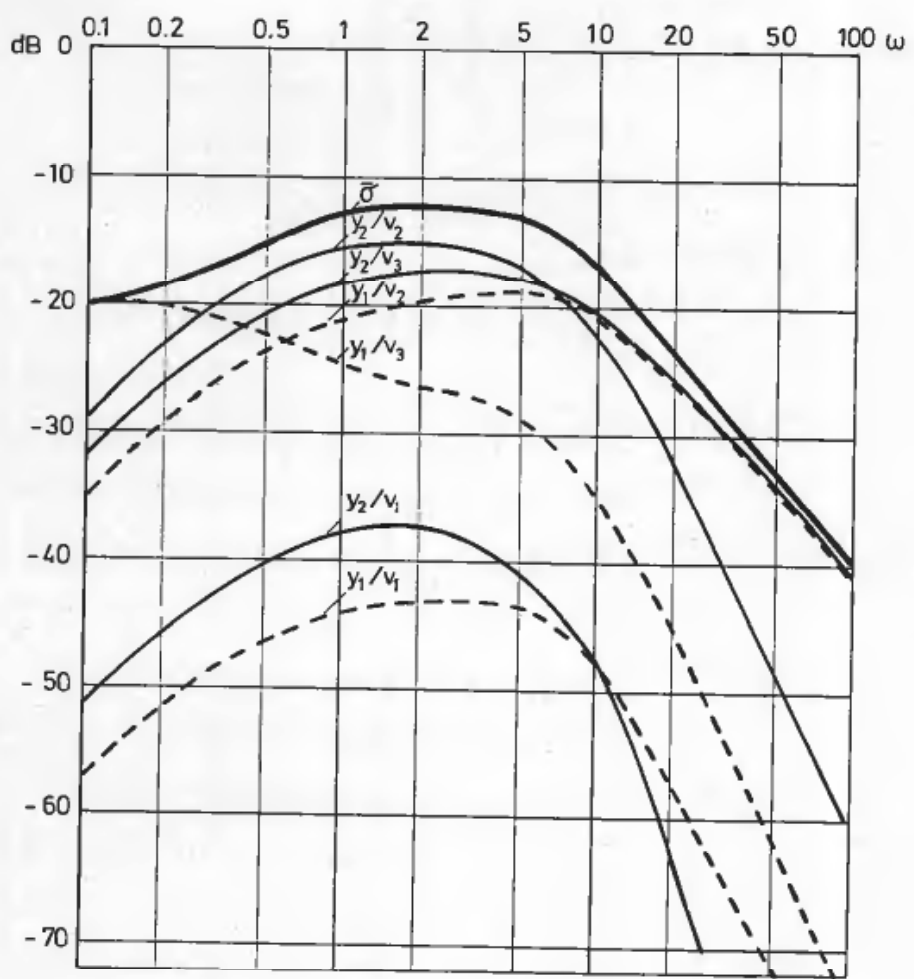

Figure 7. Example 4.

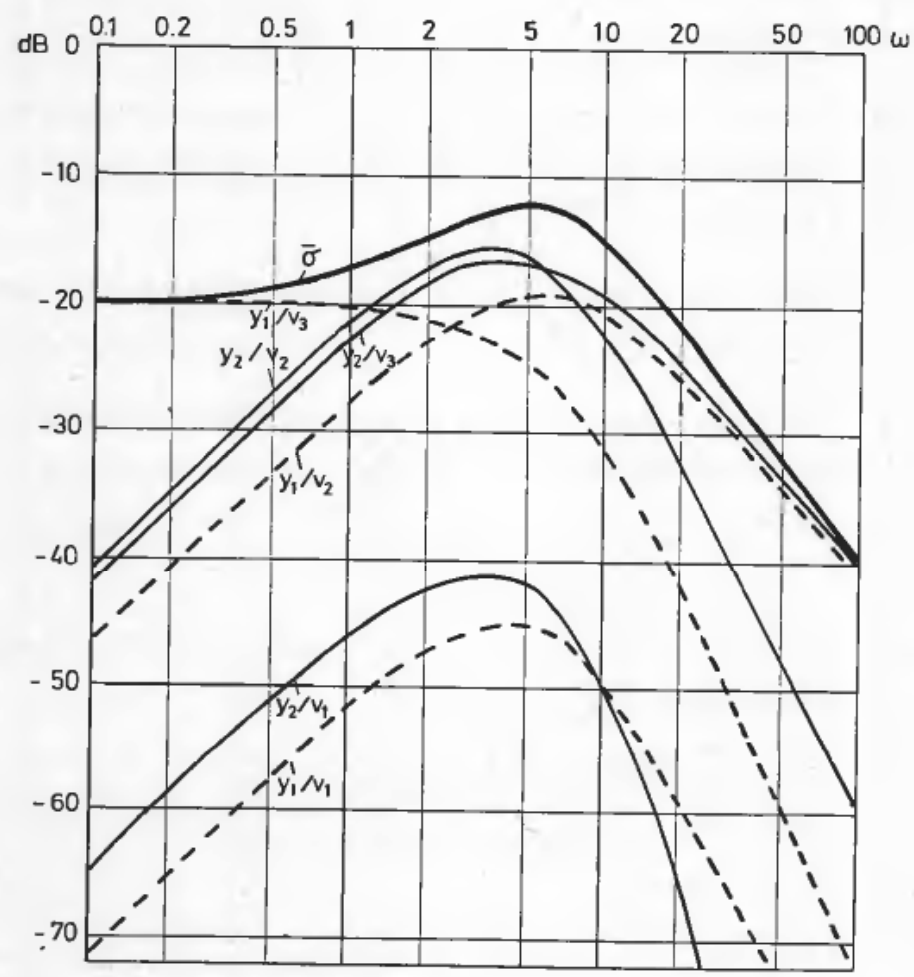

Figure 8. Example 5. 


$$
\begin{aligned}
& G_{1}=\left[\begin{array}{lll}
-5.94 & -2.64 & -1.53 \\
-0.53 & -7.3 & -2.25
\end{array}\right] \\
& G_{2}=\left[\begin{array}{l}
-4.09 \\
-10.13
\end{array}\right]
\end{aligned}
$$

The frequency response curves together with the $\bar{\sigma}$-plot are shown in Fig. 8. Compared with the previous example, Fig. 7, we have now improved the low frequency performance for all curves, except for $y_{1} / v_{3}$.

\section{Proportional feedback with state estimation}

See Fig. 9.

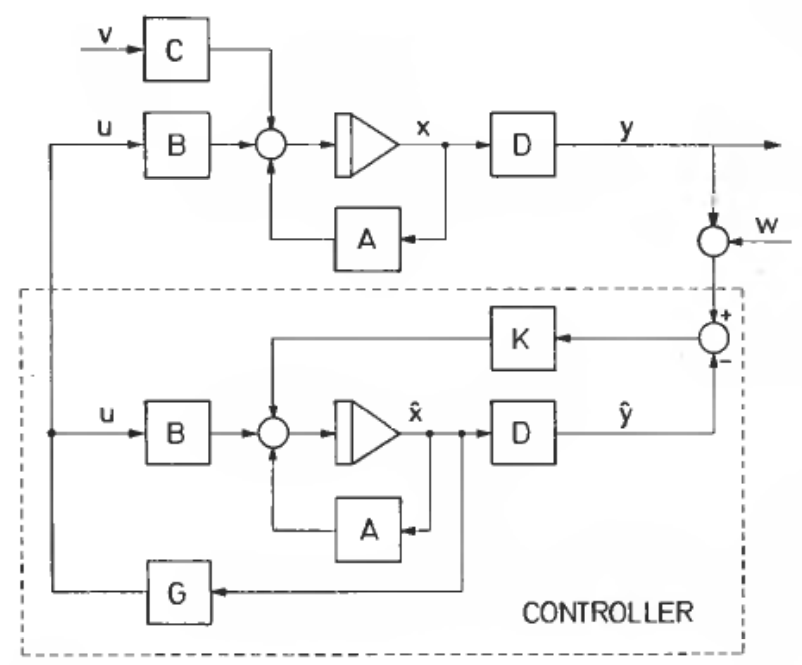

Figure 9. Proportional feedback with state estimation.

The total system is described by

$$
\begin{aligned}
{\left[\begin{array}{c}
\dot{x} \\
\hdashline \hat{x}
\end{array}\right] } & =\left[\begin{array}{c:c}
A & B G \\
\hdashline K D & A+B G-K D
\end{array}\right]\left[\begin{array}{c}
x \\
\hdashline \hat{x}
\end{array}\right]+\left[\begin{array}{c}
C \\
\hdashline 0_{n \times n}
\end{array}\right] v \\
& =\tilde{A} \tilde{x}+\tilde{C} v
\end{aligned}
$$

where $\hat{x}$ is the estimated state and $\tilde{x}$ the augmented state.

Fortunately, the design problem can be divided into two independent parts, since we can design the feedback system $(A+B G)$ independently of the estimator $(A-K D)$. For the estimator design there are several possibilities, as for example an optimal estimator (Kalman filter) or a modal type, based on eigenvalue placement. The design of an optimal estimator is based on a stochastic description of process disturbance and sensor noise. As we are considering more deterministic signals here, such a design is not directly applicable. It is, however, possible to modify the design by combining an 
optimal and a modal estimator. Let us assume that we have some knowledge about the sensor noise, so that we can specify the noise covariance matrix $W$. If we now in addition specify the eigenvalues of the estimator closed-loop matrix $(A-K D)$, we may use the same technique as for designing optimal feedback systems with specified stability, since these are dual problems (Solheim 1972). The result is not directly an optimal estimator but an estimator with prescribed stability that weighs the measurements according to their noise contents.

The matrices in the standard transfer matrix (6) are in this case

$\tilde{A}$ and $\tilde{C}$ as given in (16).

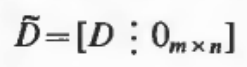

\section{Example 6}

We use the same feedback controller as in Example 1. We specify the sensor noise covariance matrix as

$$
W=\left[\begin{array}{ll}
1 & 0 \\
0 & 1
\end{array}\right]
$$

which means that both measurements contain the same amount of noise.

Let us specify the eigenvalues of the estimator $(A-K D)$ as

$$
-4,-6,-7
$$

Comparing these with the eigenvalues of the feedback system

$$
-8, \quad-5 \cdot 62 \pm j 4 \cdot 56
$$

we note that they all lie in the same area.

The specified eigenvalues of the estimator may be obtained by using the process disturbance covariance matrix

$$
V=\left[\begin{array}{ccc}
25 \cdot 6 & 16 \cdot 6 & 20 \cdot 8 \\
16 \cdot 6 & 16 \cdot 7 & 30 \cdot 1 \\
20 \cdot 8 & 30 \cdot 1 & 74 \cdot 3
\end{array}\right]
$$

We may consider this matrix as a description of some sort of fictional process disturbances.

The estimator gain matrix becomes

$$
K=\left[\begin{array}{cc}
2.15 & 2.54 \\
1.8 & 2.9 \\
2.9 & 7.2
\end{array}\right]
$$

The frequency response curves of the transfer matrix $H_{y v}(s)$ are shown in Fig. 10 together with the $\bar{\sigma}$-plot.

Comparing with Example 1, Fig. 2, which is the same system without estimator, we note that the performance has deteriorated. We will therefore try a new estimator that is somewhat faster than the feedback system itself. 


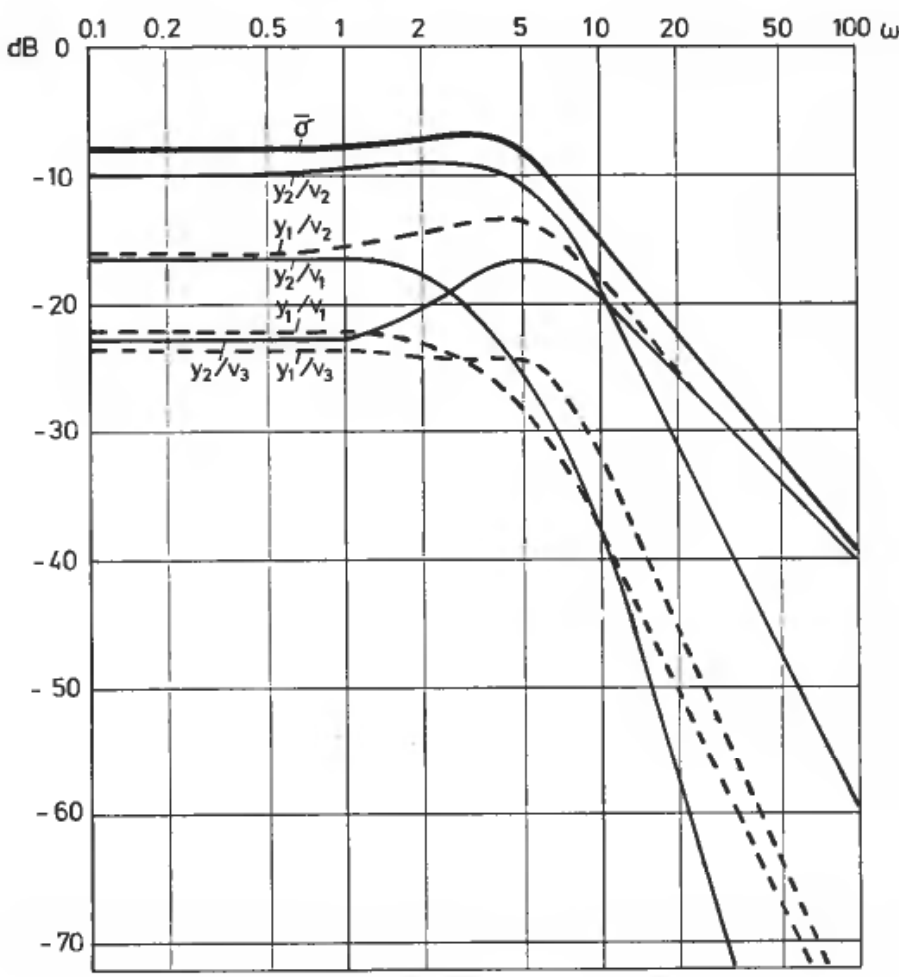

Figure 10. Example 6.

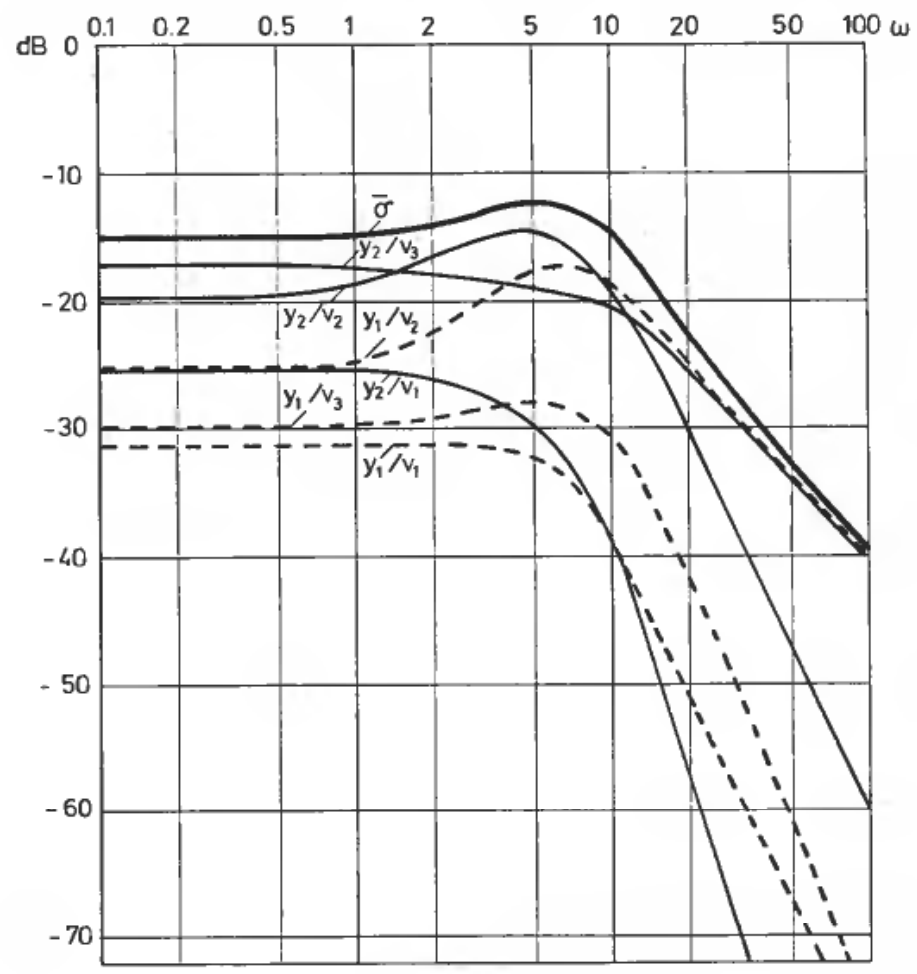

Figure 11. Example 7. 


\section{Example 7}

We specify the eigenvalues of the estimator as

$$
-10,-12,-15
$$

Comparing again with the eigenvalues of the feedback system

$$
-8, \quad-5 \cdot 62 \pm j 4 \cdot 56
$$

we note that the estimator is now somewhat faster than the feedback system.

We get the covariance matrix

and estimator gain matrix

$$
V=\left[\begin{array}{ccc}
6773 & 1228 & 398 \\
1228 & 295 & 151 \\
398 & 151 & 164
\end{array}\right]
$$

$$
K=\left[\begin{array}{cc}
68 \cdot 7 & 22 \cdot 8 \\
17 \cdot 0 & 9 \cdot 5 \\
9 \cdot 5 & 12 \cdot 0
\end{array}\right]
$$

The frequency response curves and $\bar{\sigma}$-plot are shown in Fig. 11. We note that the performance has now improved from the previous example and is not very much inferior to the case without estimator, Fig. 2.

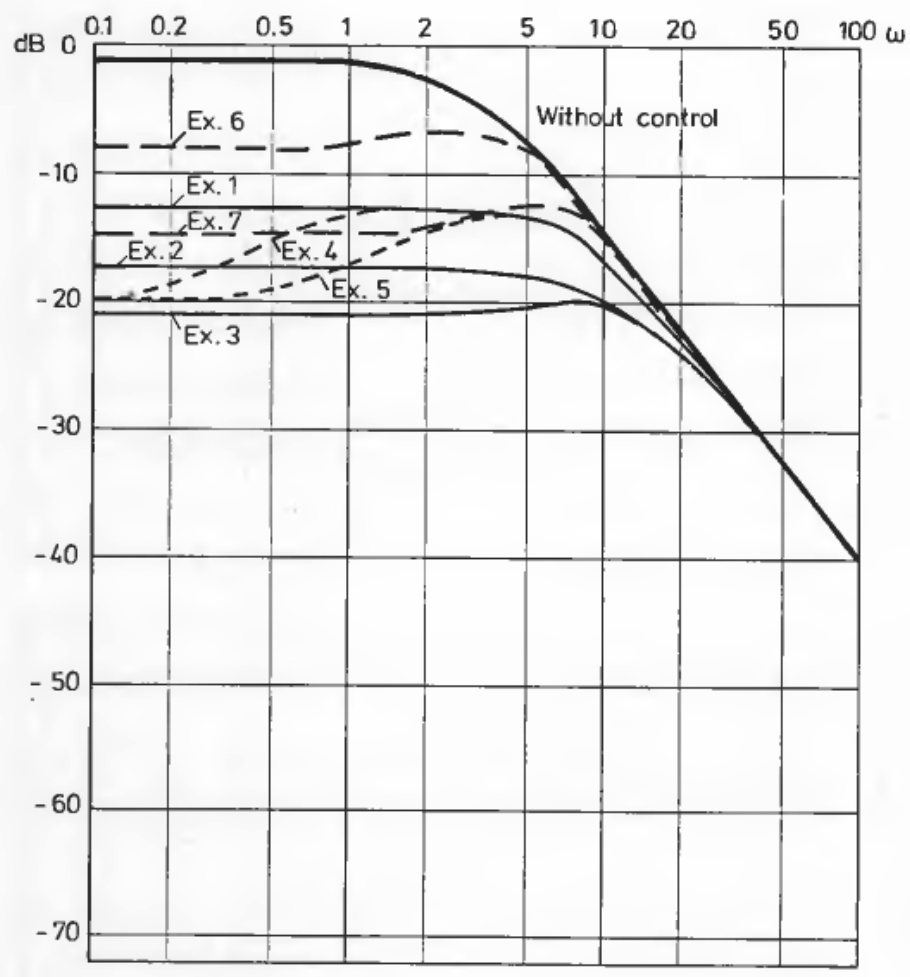

Figure 12. $\quad \tilde{\sigma}$-plots for all examples. 


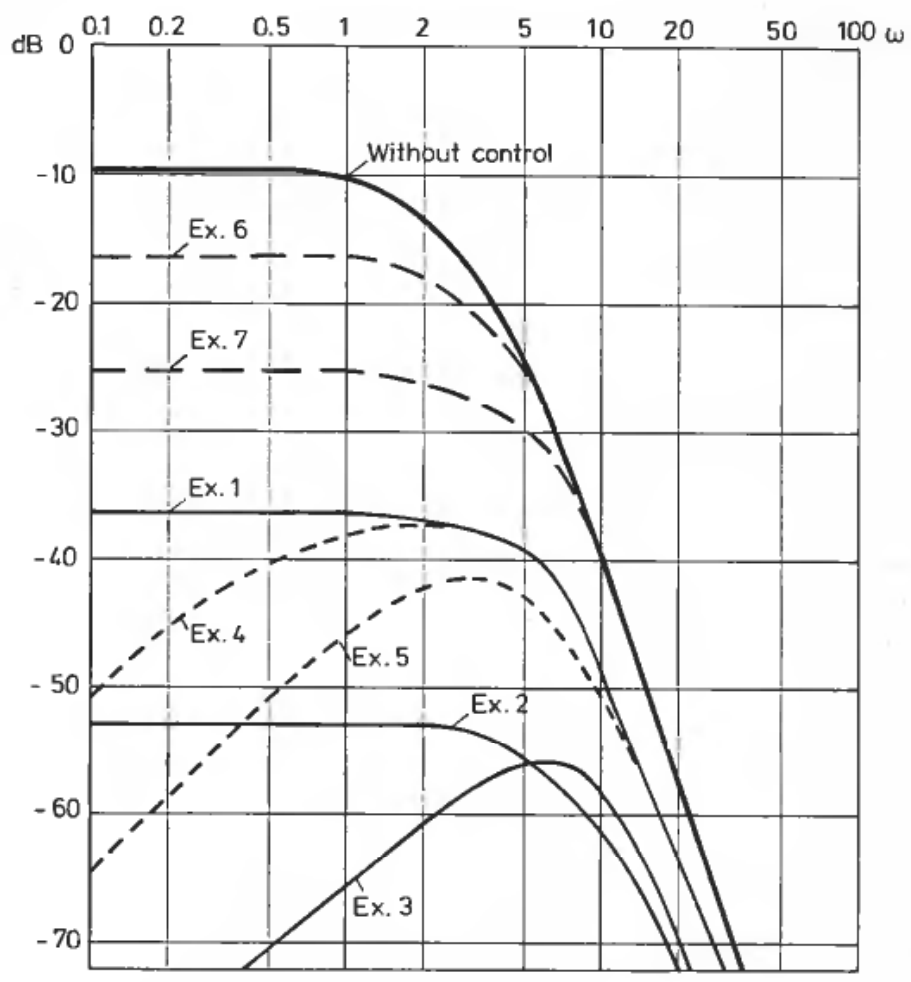

Figure 13. $y_{2} / v_{1}$-plots for all examples.

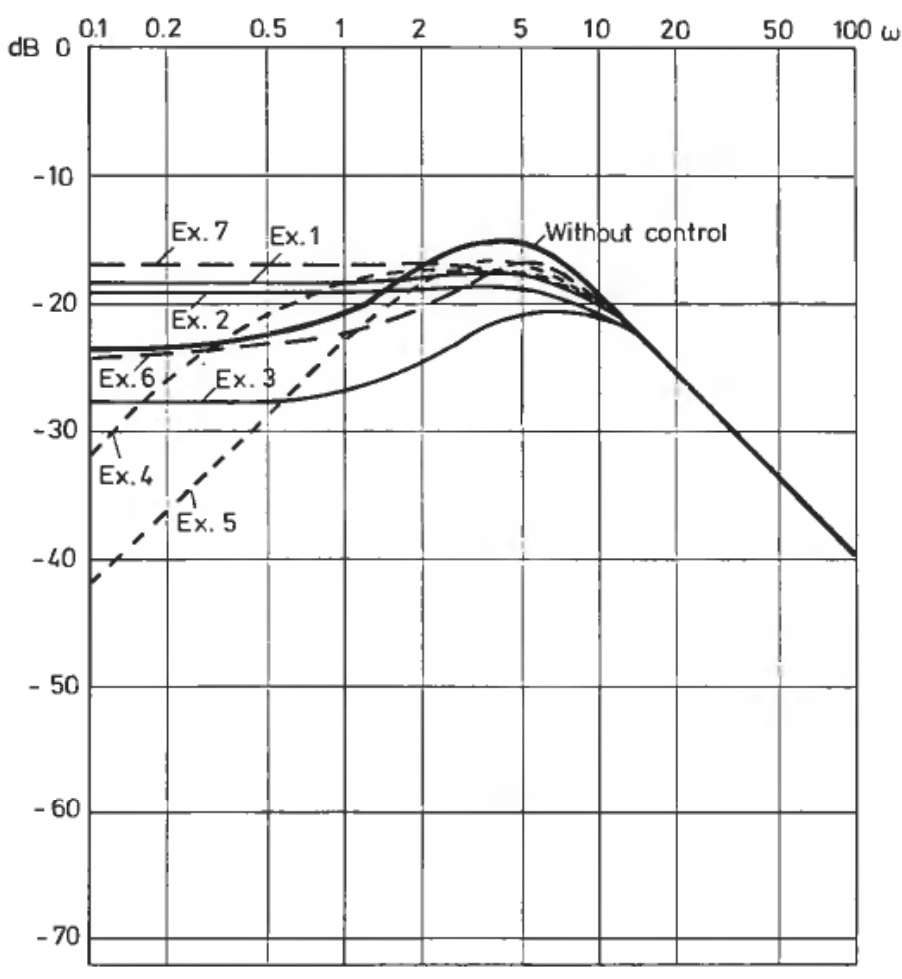

Figure 14. $y_{2} / v_{3}$-plots for all examples. 


\section{Concluding remarks}

In order to compare the different cases more easily, all the $\vec{\sigma}$-plots are shown in Fig. 12, all the $y_{2} / v_{1}$-plots in Fig. 13 and all the $y_{2} / v_{3}$-plots in Fig. 14. In addition we have also shown the case without control in these figures.

The design technique is fairly straightforward and can be adapted to different controller configurations. The frequency analysis is made easier by putting all the different configurations into a standard framework.

The $\bar{\sigma}$-plots give a useful evaluation of the performance of the system, although there are some weaknesses in using such overall descriptions. A better evaluation can therefore be obtained by also including curves that show how the individual disturbance variables influence the different output variables.

In producing the above results we have used the computer-aided systems DAREK (Pedersen, Pøhner and Solheim 1972) and CYPROS (Tyssø 1980) at the Division of Engineering Cybernetics, The Norwegian Institute of Technology.

\section{REFERENCES}

Doyle, J. C., and Stein, G. (1981). Multivariable feedback design: Concepts for a classical/ modern synthesis. I.E.E.E. Trans. Autom. Control, AC-26, 4-16.

KÜMmel, M., and OHRT, E. (1981). Practical evaluation of geometric control. 8 I.F.A.C. Congress, Kyoto, Aug. 24-28, 1981.

MacFarlane, A. G. J. (1981). Characteristic and principal gains and phases and their use as multivariable control design tools. AGARD Lecture Series No. 117, Paper No. 2, 1-34.

Pedersen, J. O., Pøhner, F., and Solneim, O. A. (1972). Computer aided design of multivariable control systems. 5. IFAC Congress, Paris, June 1972.

Solneim, O. A. (1972). Design of optimal control systems with prescribed eigenvalues. Int. J. Contr., 15, 143-160.

Solneim, O. A. (1980). On the use of low-order Riccati equations in the design of a class of feedback controllers and estimators. MIC, 1, 231-245.

Takamatsu, T., Hashimoto, I., and NaKal, Y. (1979). A geometric approach to multivariable control system design of a distillation column. Automatica, 15, 387-402.

TYssø, A. (1980). CYPROS-cybernetic program packages. MIC, 1, 215-229.

WonHAM, W. M. (1979). Geometric state-space theory in linear multivariable control: A status report. Automatica, 15, 5-13. 